Pacific Journal of Mathematics

WEAK AND NORM APPROXIMATE IDENTITIES ARE
DIFFERENT 


\title{
WEAK AND NORM APPROXIMATE IDENTITIES ARE DIFFERENT
}

\author{
Charles A. Jones and Charles D. Lahr
}

An example is given of a convolution measure algebra which has a bounded weak approximate identity, but no norm approximate identity.

1. Introduction. Let $A$ be a commutative Banach algebra, $A^{\prime}$ the dual space of $A$, and $\Delta A$ the maximal ideal space of $A$. A weak approximate identity for $A$ is a net $\{e(\lambda): \lambda \in \Lambda\}$ in $A$ such that

$$
\chi(e(\lambda) a) \longrightarrow \chi(a)
$$

for all $a \in A, \chi \in \Delta A$. A norm approximate identity for $\mathrm{A}$ is a net $\{e(\lambda): \lambda \in \Lambda\}$ in $A$ such that

$$
\|e(\lambda) a-a\| \longrightarrow 0
$$

for all $a \in A$. A net $\{e(\lambda): \lambda \in \Lambda\}$ in $A$ is bounded and of norm $M$ if there exists a positive number $M$ such that $\|e(\lambda)\| \leqq M$ for all $\lambda \in \Lambda$.

It is well known that if $A$ has a bounded weak approximate identity for which $f(e(\lambda) a) \rightarrow f(\alpha)$ for all $f \in A^{\prime}$ and $a \in A$, then $A$ has a bounded norm approximate identity [1, Proposition 4, page 58]. However, the situation is different if weak convergence is with respect to $\Delta A$ and not $A^{\prime}$. An example is given in $\S 2$ of a Banach algebra $A$ which has a weak approximate identity, but does not have a norm approximate identity. This algebra provides a counterexample to a theorem of J. L. Taylor [4, Theorem 3.1], because it is proved in [3, Corollary 3.2] that the structure space of a convolution measure algebra $A$ has an identity if and only if $A$ has a bounded weak approximate identity of norm one.

2. The example. Throughout this paper the set of complex numbers is denoted $\boldsymbol{C}$ and the set of real numbers $\boldsymbol{R}$.

Let $S$ be a commutative semigroup, and $\ell_{1}(S)$ the Banach space of all complex functions $\alpha: S \rightarrow C$ such that $\|\alpha\|=\sum_{x \in S}|\alpha(x)|$ is finite, made into a convolution algebra under the product

$$
\alpha * \beta=\sum_{x \in S} \sum_{\substack{u, v \\ u v=x}} \alpha(u) \beta(v) \delta_{x},
$$

where $\delta_{x}$ represents the point mass at $x \in S, \alpha=\sum_{x \in S} \alpha(x) \delta_{x}$ and $\beta=\sum_{x \in S} \beta(x) \delta_{x}$. A semicharacter on $S$ is a bounded nonzero function $\chi: S \rightarrow C$ such that $\chi(x y)=\chi(x) \chi(y)$ for all $x, y \in S$. The set of 
all semicharacters is denoted $\hat{S}$.

It has been shown in a previous paper [3] that if $\ell_{1}(S)$ is semisimple, then the existence of a bounded weak approximate identity of norm one in $\ell_{1}(S)$ is equivalent to the existence of a net $\left\{u_{d}\right\}$ in $S$ such that $\chi\left(u_{d}\right) \rightarrow 1$ for all $\chi \in \widehat{S}$. It has also been shown that the existence of a norm approximate identity bounded by 1 is equivalent to the existence of a net $\left\{u_{d}\right\}$ in $S$ with the following property: for each $x \in S$, there exists $d_{x}$ such that $x u_{d}=x$ for all $d \geqq d_{x}$. For the particular semigroup $S$ to follow, it will be shown that $\ell_{1}(S)$ does indeed have a bounded weak approximate identity, but does not have a norm approximate identity.

Let the set of integers be denoted by $Z$ and the set of positive integers by $\boldsymbol{Z}^{+}$. Further, let $S=\left\{m / n: m, n \in \boldsymbol{Z}^{+}\right\}$under addition. Then $S$ is a cancellative semigroup and so $\ell_{1}(S)$ is semisimple [2]. If $\chi \in \hat{S}$, then $\chi$ is uniquely determined by its values on $\left\{1 / n: n \in \boldsymbol{Z}^{+}\right\}$. For if $m$ is any positive integer, then for all $n \in Z^{+}, \chi(m / n)=\chi(1 / n)^{m}$. In fact $\chi(1)=\chi(n / n)=\chi(1 / n)^{n}$ for all $n \in Z^{+}$, and so $\chi(1 / n)$ is an $n$th root of $\chi(1)$. Now, each pair $(k, z)$, where $k \in Z$ and $z=r e^{i \theta}$ with $|z| \leqq 1$ and $r, \theta \in \boldsymbol{R}$, determines a semicharacter $\chi_{k, z}$ of $S$ by defining

$$
\chi_{k, z}(m / n)=r^{m / n} e^{i m(\theta+2 k \pi) / n}
$$

for all $m / n$ in $S$. It is clear that $\chi_{k, z}(1 / n) \rightarrow 1$ for each $\chi_{k, z} \in \hat{S}$. However, not all semicharacters have such a nice form. In constructing an arbitrary semicharacter $\chi$, there are very few restrictions imposed upon how the $n$th root of $\chi(1)$ is to be chosen. Thus, a more elaborate argument is required to obtain a weak approximate identity for $\ell_{1}(S)$.

LEMma 2.1. Let $G$ be an infinite discrete group with identity $e$. Then there exists a net $\left\{g_{\lambda}\right\} \subset G, g_{\lambda} \neq e$ for all $\lambda$, such that $\chi\left(g_{\lambda}\right) \rightarrow 1$ for each $\chi \in \hat{G}$.

Proof. Let $\bar{G}$ be the Bohr compactification of $G$. Then there is an algebra isomorphism $i$ of $G$ onto a dense subset of $\bar{G}$. Specifically, for each $g \in \bar{G}$, there exists a net $\left\{i\left(g_{\lambda}\right): g_{\lambda} \in G\right\}$ such that $i\left(g_{\lambda}\right) \rightarrow g$; equivalently, $\bar{\chi}\left(i\left(g_{\lambda}\right)\right) \rightarrow \bar{\chi}(g)$ for each $\chi \in \hat{G}$, where $\bar{\chi}$ is the unique extension of $\chi \in \hat{G}$ to $\bar{\chi} \in \hat{\bar{G}}$ [3]. Since $\bar{G}$ is infinite and compact, the identity $i(e)$ of $\bar{G}$ is not isolated in $\bar{G}$. Hence, there is a net $\left\{i\left(g_{\lambda}\right): g_{\lambda} \in G\right\}, g_{\lambda} \neq e$ for all $\lambda$, such that $i\left(g_{\lambda}\right) \rightarrow i(e)$. Therefore,

$$
\chi\left(g_{\lambda}\right)=\bar{\chi}\left(i\left(g_{\lambda}\right)\right) \longrightarrow \bar{\chi}(i(e))=1 \text { for each } \chi \in \hat{G} \text {. }
$$

Let $T=\{z \in C:|z|=1\}$ and $D=\{z \in C:|z| \leqq 1\}$. Then the previous lemma yields the following number-theoretic result. 
THEOREM 2.2. Let $\left\{z_{1}, z_{2}, \cdots, z_{p}\right\} \subset T, p \in Z^{+}$. Then for each $\varepsilon>0$ there exists $m \in \boldsymbol{Z}^{+}$such that $\left|\left(\boldsymbol{z}_{i}\right)^{m}-1\right|<\varepsilon$ for all $i, 1 \leqq$ $i \leqq p$.

Proof. Consider the group $G=\boldsymbol{Z}$ under addition. Then $\hat{G}=$ $\left\{\chi_{z}: z \in T\right\}$, where $\chi_{z}(n)=z^{n}, n \in G$. Now, let $\varepsilon>0$ be given. By Lemma 2.1, there exists a net $\left\{n_{\lambda}: \lambda \in \Lambda\right\} \subset G, n_{\lambda} \neq 0$ for all $\lambda$, such that $z^{n_{\lambda}}=\chi_{z}\left(n_{\lambda}\right) \rightarrow 1$ for each $\chi_{z} \in \hat{G}$. Without loss of generality, assume that $n_{\lambda} \in \boldsymbol{Z}^{+}$for all $\lambda$. Hence, given $\left\{z_{1}, z_{2}, \cdots, z_{p}\right\} \subset T$, there exist $\lambda_{1}, \lambda_{2}, \cdots, \lambda_{p}$ in $A$ such that $\left|z_{i}^{n} \lambda-1\right|<\varepsilon$ for all $\lambda \geqq \lambda_{i}, 1 \leqq$ $i \leqq p$. Thus, if $\lambda_{0} \in A$ is such that $\lambda_{0} \geqq \lambda_{i}, 1 \leqq i \leqq p$, then with $m=n_{\lambda_{0}}$,

$$
\left|\left(z_{i}\right)^{m}-1\right|<\varepsilon \text { for } i=1,2, \cdots, p
$$

Corollary 2.3. Let $\left\{z_{1}, z_{2}, \cdots, z_{p}\right\} \subset T, p \in \boldsymbol{Z}^{+}$. Then for each $\varepsilon, 0<\varepsilon<1$, there exist neighborhoods $U_{1}, U_{2}, \cdots, U_{p}$ and there exists $m_{0} \in Z^{+}$such that

(1) $z_{i} \in U_{i}$ and $U_{i} \subset D, 1 \leqq i \leqq p$,

(2) $|u-1|<\varepsilon$ for all

$$
u \in U_{i}^{m_{0}}=\left\{w_{1} w_{2} \cdots w_{m_{0}}: w_{j} \in U_{i}\right\}, \quad 1 \leqq i \leqq p .
$$

Proof. Let $z_{j}=e^{i \theta_{j}}, 1 \leqq j \leqq p . \quad$ By Theorem 2.2, there exists $m_{0} \in \boldsymbol{Z}^{+}$such that $\left|m_{0} \theta_{j}(\bmod 2 \pi)\right|<\varepsilon / 2$ for all $j$. Now, for each $j$, let

$$
U_{j}=\left\{w=|w| e^{i \omega} \varepsilon D:\left|\omega-\theta_{j}\right|<\frac{\varepsilon}{4 m_{0}} \text { and }|w|>\left[1-\frac{\varepsilon}{4}\right]^{1 / m_{0}}\right\} .
$$

Then if $u \in U_{j}^{m_{0}}, u=w_{1} w_{2} \cdots w_{m_{0}}, w_{k} \in U_{j}$ for all $k$, so that $\mid \omega_{1}+$ $\omega_{2}+\cdots+\omega_{m_{0}}-m_{0} \theta_{j} \mid<\varepsilon / 4$ and $\left|w_{1}\right|\left|w_{2}\right| \cdots\left|w_{m_{0}}\right|>1-\varepsilon / 4$. Thus, if $u \in U_{j}^{m_{0}}$, then

$$
|u-1| \leqq\left|u-z_{j}^{m_{0}}\right|+\left|z_{j}^{m_{0}}-1\right|<\frac{\varepsilon}{2}+\frac{\varepsilon}{2}=\varepsilon .
$$

After a technical lemma, the desired result will be proved. $S$ continues to be the semigroup of positive rationals under addition.

Lemma 2.4. Let $\left\{\chi_{1}, \chi_{2}, \cdots, \chi_{p}\right\} \subset \hat{S}, p \in Z^{+}$. Then there exists $a$ subsequence $\left\{1 / n_{k}: k \in Z^{+}\right\}$of $\left\{1 / n: n \in Z^{+}\right\}$and there exist $z_{1}, z_{2}, \cdots$, $z_{p} \in T$ such that $\chi_{i}\left(1 / n_{k}\right) \rightarrow z_{i}$ for each $i, 1 \leqq i \leqq p$.

Proof. Note that for each $i, \chi_{i}(1 / n)$ is an $n$th root of $\chi_{i}(1)$ and so $\left|\chi_{i}(1 / n)\right| \rightarrow 1$ as $n \rightarrow \infty$. 
Now, $\left\{\chi_{1}(1 / n): n \in Z^{+}\right\}$is a subset of the closed unit disk $D$, and so by compactness has a convergent subsequence with limit $z_{1} ; z_{1} \in T$ by the above remark. Further, if a subsequence $\left\{1 / n_{\ell}: \ell \in Z^{+}\right\}$exists such that $\chi_{i}\left(1 / n_{\ell}\right) \rightarrow z_{i}$ for $i=1,2, \cdots, j$, then by compactness $\left\{\chi_{j+1}\left(1 / n_{\ell}\right): \ell \in Z^{+}\right\}$has a convergent subsequence $\left\{\chi_{j+1}\left(1 / n_{k}\right): k \in Z^{+}\right\}$ with limit $z_{j+1} \in T$. Thus, the induction proof is complete.

THEOREM 2.5. There exists a net $\left\{q_{d}: d \in \mathscr{D}\right\} \subset S$ such that $\chi\left(q_{d}\right) \rightarrow 1$ for each $\chi \in \hat{S}$. Therefore, $\ell_{1}(S)$ has a weak approximate identity of norm one.

Proof. Let $\mathscr{F}(\hat{S})$ denote the collection of all finite subsets of $\hat{S}$ and let $\mathscr{D}=Z^{+} \times \mathscr{F}(\hat{S})$ be directed by $(n, A) \leqq(m, B)$ if and only if $n \leqq m$ and $A \subset B$.

Now, define a mapping $d \mapsto q_{d}$ of $\mathscr{D}$ into $S$ as follows: For each $d=(n, A), A=\left\{\chi_{1}, \cdots, \chi_{p}\right\}$, fix a subsequence $\left\{1 / n_{k}: k \in Z^{+}\right\}$such that $\chi_{i}\left(1 / n_{k}\right) \rightarrow z_{i} \in T$ for all $i$. Then there exist $m_{0} \in Z^{+}$and neighborhoods $U_{1}, \cdots, U_{p}$ of $z_{1}, \cdots, z_{p}$, respectively, such that $|u-1|<1 / n$ for all $u \in U_{j}^{m_{0}}, 1 \leqq i \leqq p$. Now, there exist $K_{i} \in Z^{+}$such that $k \geqq K_{i}$ implies $\chi_{i}\left(1 / n_{k}\right) \in U_{i}$ for $1 \leqq i \leqq p$. Hence, for each $i, 1 \leqq i \leqq p$,

$$
\left|\chi_{i}\left(m_{0} / n_{k}\right)-1\right|=\left|\chi_{i}\left(1 / n_{k}\right)^{m_{0}}-1\right|<\frac{1}{n}
$$

for all $k \geqq K_{i}$. Set $K_{0}=\max \left\{K_{i}: i=1,2, \cdots, p\right\}$. Then define $q_{d}=$ $m_{0} / n_{K_{0}}$.

Finally, it remains to show that for each $\chi \in \hat{S}, \chi\left(q_{d}\right) \rightarrow 1$. So, let $\varepsilon>0$ be given. Then choose $n_{0}$ such that $\left(1 / n_{0}\right)<\varepsilon$, and let $A_{0}=\{\chi\}$. If $d=(n, A) \geqq\left(n_{0}, A_{0}\right)=d_{0}$, then $\left|\chi\left(q_{d}\right)-1\right|<\left(1 / n_{0}\right)<\varepsilon$.

COROLLARY 2.6. There exists a net $\left\{1 / n_{d}: d \in \mathscr{D}\right\} \subset\left\{1 / n: n \in Z^{+}\right\}$ such that $\chi\left(1 / n_{d}\right) \rightarrow 1$ for each $\chi \in \hat{S}$.

Proof. Repeat the proofs of Lemma 2.4 and Theorem 2.5 with $\left\{1 / n: n \in Z^{+}\right\}$replaced by $\left\{1 / n !: n \in Z^{+}\right\}$. Then in the proof of Theorem 2.5 choose $K_{0}$ such that

(1) $K_{0} \geqq \max \left\{K_{i}: i=1,2, \cdots, p\right\}$ and

(2) $n_{K_{0}} \geqq m_{0}$. Thus, $q_{d}=m_{0} / n_{K_{0}}$ ! is of the form $1 / n_{d}$ for some $n_{d} \in \boldsymbol{Z}^{+}$.

Theorem 2.5 and Corollary 2.6 make it clear that $\ell_{1}(S)$ has a bounded weak approximate identity $\left\{\delta_{1 / n_{d}}: d \in \mathscr{D}\right\}$ [3]. However, $S$ does not have relative units. That is, given $m / n \in S$, there is no $v \in S$ such that $v(m / n)=m / n$. Thus, $l_{1}(S)$ does not have a norm approximate identity, bounded or unbounded. 
3. A general result. The same techniques developed in $\S 2$ can be used to prove a useful result about weak approximate identities of norm one for a commutative Banach algebra.

THEOREM 3.1. Let $A$ be a commutative Banach algebra. Then $A$ has a weak approximate identity of norm one if and only if there exists a net $\{v(\rho): \rho \in \mathscr{F}\}$ in $A$, $\|v(\rho)\| \leqq 1$ for all $\rho$, such that $|\chi(v(\rho))| \rightarrow 1$ for all $\chi \in \Delta A$.

Proof. If $A$ has a weak approximate identity of norm one, then there exists a net $\{v(\rho): \rho \in \mathscr{J}\}$ in $A,\|v(\rho)\| \leqq 1$ for all $\rho$, such that

$$
\chi(v(\rho) a) \longrightarrow \chi(a) \text { for all } a \in A, \chi \in \Delta A .
$$

Thus, for each $\chi \in \Delta A, \chi(a) \neq 0$ for some $a \in A$ implies that $\chi(v(\rho)) \rightarrow 1$ and hence $|\chi(v(\rho))| \rightarrow 1$.

Conversely, assume that $\{v(\rho)\}$ is such that $|\chi(v(\rho))| \rightarrow 1$ for each $\chi \in \Delta A$. Let $\mathscr{F}(\Delta A)$ be the collection of all finite subsets of $\Delta A$ and let $\Lambda=Z^{+} \times \mathscr{F}(\Delta A)$ be directed by $(n, F) \leqq(m, E)$ if and only if $n \leqq m$ and $F \subset E$.

Then define a mapping $\lambda \mapsto e(\lambda)$ of $\Lambda$ into $A$ as follows: for each $\lambda=(n, F)$, where $n \in Z^{+}$and $F=\left\{\chi_{1}, \chi_{2}, \cdots, \chi_{r}\right\}$, there exists by compactness of $D$ a subnet $\left\{v\left(\rho^{\prime}\right)\right\}$ of $\{v(\rho)\}$ such that $\chi_{i}\left(\rho^{\prime}\right) \rightarrow z_{i} \in T$ for $i, 1 \leqq i \leqq r$. By Corollary 2.3, there exists $m_{0} \in Z^{+}$and neighborhoods $U_{i}$ of $z_{i}$ in $D$ such that $|z-1|<1 / n$ for all $z \in U_{i}^{m_{0}}, 1 \leqq$ $i \leqq r$. Now, let $\rho_{0}^{\prime}$ be such that $\chi_{i}\left(v\left(\rho_{0}^{\prime}\right)\right) \in U_{i}$ for all $i, 1 \leqq i \leqq r$, and define $e(\lambda)=v\left(\rho_{0}^{\prime}\right)^{m_{0}}$. Note that for each $i$,

$$
\begin{aligned}
\left|\chi_{i}(e(\lambda))-1\right| & =\left|\chi_{i}\left(v\left(\rho_{0}^{\prime}\right)^{m_{0}}\right)-1\right| \\
& =\left|\left(\chi_{i}\left(v\left(\rho_{0}^{\prime}\right)\right)\right)^{m_{0}}-1\right|<\frac{1}{n} .
\end{aligned}
$$

Thus, $\chi(e(\lambda)) \rightarrow 1$ for each $\chi \in \Delta A$ and hence $\chi(e(\lambda) \alpha) \rightarrow \chi(a)$ for each $\chi \in \Delta A, a \in A$. Also, $\|e(\lambda)\|=\left\|v\left(\rho_{0}^{\prime}\right)^{m_{0}}\right\| \leqq 1$ for all $\lambda \in \Lambda$.

CoROLlaRY 3.2. Let $S$ be a commutative semigroup for which $\ell_{1}(S)$ is semisimple. Then $\ell_{1}(S)$ has a weak approximate identity of norm one if and only if there exists a net $\{s(\rho):(\rho) \in \mathscr{J})\}$ in $S$ such that $|\chi(s(\rho))| \rightarrow 1$ for all $\chi \in \hat{S}$.

Proof. The Banach algebra $\ell_{1}(S)$ has a weak approximate identity of norm one if and only if there exists a net $\{s(\lambda): \lambda \in \Lambda\}$ in $S$ such that $\chi(s(\lambda)) \rightarrow 1$ for all $\chi \in \hat{S}$ [3]. Thus, the proof is completed by applying Theorem 3.1 with $v(\rho)=\delta_{s(\rho)}$ for all $\rho$. 


\section{REFERENCES}

1. F. F. Bonsall and J. Duncan, Complete Normed Algebras, Springer-Verlag, 1973.

2. E. Hewitt and H. S. Zuckerman, The $\ell_{1}$-algebra of a commutative semigroup, Trans. Amer. Math. Soc., 83 (1956), 70-97.

3. C. D. Lahr, Approximate identities for convolution measure algebras, Pacific J. Math., 47 (1973), 147-159.

4. J. L. Taylor, The structure of convolution measure algebras, Trans. Amer. Math. Soc., 119 (1965), 150-166.

Received January 25, 1977.

Dartmouth College

HANOVER, NH 03755 


\title{
PACIFIC JOURNAL OF MATHEMATICS
}

\author{
EDITORS
}

RICHARD ARENS (Managing Editor)

University of California

Los Angeles, California 90024

C. W. CurTis

University of Oregon

Eugene, OR 97403

C. C. MOORE

University of California

Berkeley, CA 94720
J. DUgundJI

Department of Mathematics

University of Southern Californı

Los Angeles, California 90007

R. Finn and J. Milgram

Stanford University

Stanford, California 94305

\section{ASSOCIATE EDITORS}

E. F. BECKENBACH

\section{SUPPORTING INSTITUTIONS}

\author{
UNIVERSITY OF BRITISH COLUMBIA \\ CALIFORNIA INSTITUTE OF TECHNOLOGY \\ UNIVERSITY OF CALIFORNIA \\ MONTANA STATE UNIVERSITY \\ UNIVERSITY OF NEVADA, RENO \\ NEW MEXICO STATE UNIVERSITY \\ OREGON STATE UNIVERSITY \\ UNIVERSITY OF OREGON \\ OSAKA UNIVERSITY
}

UNIVERSITY OF SOUTHERN CALIFORNIA

STANFORD UNIVERSITY

UNIVERSITY OF TOKYO

UNIVERSITY OF UTAH

WASHINGTON STATE UNIVERSITY

UNIVERSITY OF WASHINGTON
AMERICAN MATHEMATICAL SOCIETY
NAVAL WEAPONS CENTER 


\section{Pacific Journal of Mathematics \\ Vol. 72 , No. 1 \\ January, 1977}

Kazuo Anzai and Shiro Ishikawa, On common fixed points for several



Bruce Alan Barnes, When is a representation of a Banach $*$-algebra

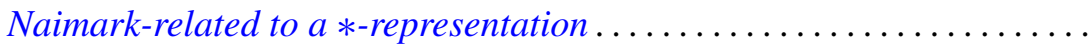

Richard Dowell Byrd, Justin Thomas Lloyd, Franklin D. Pedersen and

James Wilson Stepp, Automorphisms of the semigroup of finite

complexes of a periodic locally cyclic group ...................

Donald S. Coram and Paul Frazier Duvall, Jr., Approximate fibrations and a

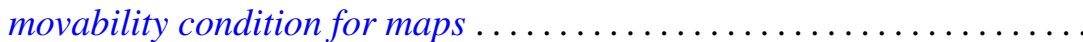

Kenneth R. Davidson and Che-Kao Fong, An operator algebra which is not

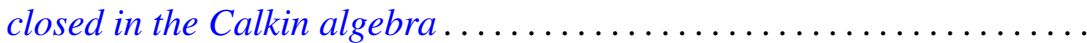

Garret J. Etgen and James Pawlowski, A comparison theorem and oscillation criteria for second order differential systems .............

Philip Palmer Green, $C^{*}$-algebras of transformation groups with smooth

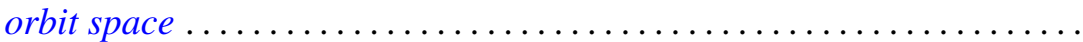

Charles Allen Jones and Charles Dwight Lahr, Weak and norm approximate

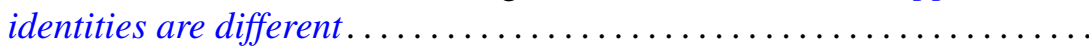

G. K. Kalisch, On integral representations of piecewise holomorphic



Y. Kodama, On product of shape and a question of Sher

Heinz K. Langer and B. Textorius, On generalized resolvents and

$Q$-functions of symmetric linear relations (subspaces) in Hilbert

space ...................................

Albert Edward Livingston, On the integral means of univalent, meromorphic functions

Wallace Smith Martindale, III and Susan Montgomery, Fixed elements of

Jordan automorphisms of associative rings ..........

R. Kent Nagle, Monotonicity and alternative methods for nonlinear boundary value problems ........................

Richard John O'Malley, Approximately differentiable functions: the $r$ topology.

Mangesh Bhalchandra Rege and Kalathoor Varadarajan, Chain conditions

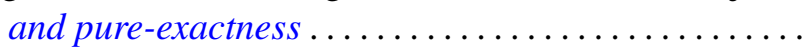

Christine Ann Shannon, The second dual of $C(X)$. .

Sin-ei Takahasi, A characterization for compact central double centralizers of $C^{*}$-algebras .

Theresa Phillips Vaughan, A note on the Jacobi-Perron algorithm. . .

Arthur Anthony Yanushka, A characterization of $\operatorname{PSp}(2 m, q)$ and

$\mathrm{P} \Omega(2 m+1, q)$ as rank 3 permutation groups ......... 